\title{
Linkage analysis of the glucokinase locus in familial Type 2 (non-insulin-dependent) diabetic pedigrees
}

\author{
S.C.EIbein ${ }^{1}$, M.Hoffman ${ }^{1}$, K. Chiu ${ }^{2}$, Y. Tanizawa ${ }^{2}$ and M.A.Permutt ${ }^{2}$ \\ ${ }^{1}$ Division of Endocrinology and Metabolism, Department of Internal Medicine, Veterans Affairs Medical Center and University \\ of Utah School of Medicine, Salt Lake City, Utah; \\ ${ }^{2}$ Division of Endocrinology and Metabolism, Washington University School of Medicine, St. Louis, Missouri, USA
}

\begin{abstract}
Summary. Glucokinase is among the few genes which may play a key role in both insulin secretion and insulin action. Glucokinase is present in pancreatic beta cells where it may have a key role in the glucose sensing mechanism, and it is present in hepatocytes, where it may participate in glucose flux. Glucokinase defects have recently been implicated in maturity-onset diabetes of the young. To examine the hypothesis that glucokinase plays a key role in the predisposition to common familial Type 2 (non-insulin-dependent) diabetes mellitus, we typed 399 members of 18 Utah pedigrees with multiple Type 2 diabetic individuals for two markers in the $5^{\prime}$ and $3^{\prime}$ flanking regions of the glucokinase gene. Linkage analysis was performed under both dominant and recessive models. We also repeated these analyses with individuals with impaired glucose tolerance who were considered affected if their stimulated (2-h) glucose exceeded age-specific normal levels for $95 \%$ of the population. Under
\end{abstract}

several dominant models, linkage was significantly excluded, and under recessive models $\log$ of the odds (LOD) score was less than -1 . We were also unable to demonstrate statistical support for the hypothesis that a small subgroup of pedigrees had glucokinase defects, but the most suggestive pedigree (individual pedigree LOD 1.8-1.9) ranked among the youngest and leanest in our cohort. We can exclude a major role for glucokinase in familial Type 2 diabetes, but our data cannot exclude a role for this locus in a minority of pedigrees. Further testing of the hypothesis that glucokinase defects contribute to diabetes in a small proportion of Type 2 diabetic pedigrees must await thorough sequence analysis of the glucokinase gene, including regulatory regions, particularly from pedigrees with positive LOD scores.

Key words: Familial Type 2 (non-insulin-dependent) diabetes mellitus linkage analysis, glucokinase.
The genetic predisposition to Type 2 (non-insulin-dependent) diabetes mellitus is well accepted, but the specific biochemical defect which characterizes that predisposition is uncertain. Many studies of Type 2 diabetes have identified hyperinsulineamia or insulin resistance as an early finding in the relatives of diabetic patients [1], but a second defect in insulin secretion is probably necessary for the full development of the diabetic phenotype [2,3]. This model is consistent with the interaction of several epistatic loci which was suggested by population risk ratios among relatives of diabetic patients [4]. Thus, genes from either pathways of insulin action or secretion may represent candidates for the genetic predisposition to Type 2 diabetes. Glucokinase (GCK) is among the few cloned genes which are present in both pathways. In the liver, GCK phosphorylates glucose and maintains a gradient for inward transport. In the pancreatic beta cell, GCK again determines the rate of phosphorylation and thus glucose flux [5,6]. Consequently GCK is thought to play a major role in beta-cell glucose sensing and glucose- stimulated insulin secretion. Perhaps most importantly, mutations of GCK have been implicated in affected members of French and English families with autosomal dominant early-onset Type 2 diabetes (maturity-onset diabetes of the young; MODY; [7-9]). Additionally, the polymorphisms of this locus are associated with Type 2 diabetes in the American Black population [10]. Glucokinase is the only cloned gene to our knowledge which has been implicated in a Type 2 diabetic subgroup by linkage. However, to date linkage or sequence analyses of this gene have only been reported in the uncommon MODY form of Type 2 diabetes, and GCK mutations may account for the genetic predisposition in fewer than $50 \%$ of these pedigrees.

To determine the role of GCK in the common forms of familial Type 2 diabetes, we examined 18 large pedigrees with two or more affected individuals ( 399 individuals) for linkage between glucokinase and familial Type 2 diabetes. While we can reject the GCK locus as a cause of Type 2 diabetes under models of homogeneity, our results cannot 
exclude and may suggest a minor role for glucokinase in the predisposition to familial Type 2 diabetes.

\section{Subjects and methods}

\section{Study population}

Studies were performed on members of 16 Caucasian pedigrees ascertained for two or more Type 2 diabetic siblings. These pedigrees are described in considerable detail elsewhere [11, 12], including pedigree diagrams [12]. An additional two pedigrees were ascertained for a mixture of Type 1 and Type 2 diabetes. Each of these pedigrees included at least one Type 2 diabetic sibling pair and were thus appropriate for study. Briefly, pedigrees were drawn from several sources in a uniform population representative of Northern European immigrants. Pedigrees with three or more Type 2 diabetic siblings were preferentially sampled, and pedigrees in which most affected members exceeded $50 \%$ of ideal body weight or in which both parents were known to be affected were not sampled. For each proband or sibling of the proband, available parents, spouses, and the two-four oldest offspring were sampled. Clinical studies of age of onset, obesity, and fasting or stimulated insulin levels did not reveal significant differences between mixed Type 1 and Type 2 diabetic pedigrees and those ascertained for Type 2 diabetes only (S.C.Elbein, unpublished data). In addition to measurements of height and weight, each pedigree member not known to be affected underwent a standard 75-g oral glucose tolerance test (OGTT) with both fasting and 1-h stimulated insulin levels, as described elsewhere [11]. Although the parents of the proband were deceased in 14 of 18 pedigrees, at least two generations were available for all pedigrees. None of our pedigrees met the definitions of MODY used in other studies. All individuals reported in previous studies [12] were included in this analysis.

\section{Disease status}

Individuals with Type 1 diabetes (onset before age 20, insulintreated) were considered of unknown status with regard to Type 2 diabetes. All individuals under pharmacological treatment for Type 2 diabetes were considered affected. We classified untreated individuals who met World Health Organization (WHO) criteria for diabetes only by 2 -h-stimulated glucose on OGTT as unknown unless the stimulated glucose level exceeded the age-and race-specific $95 \%$ levels reported from NHANES data [13]. This requirement resulted in the classification of three individuals over age 70 years with 2-h-stimulated glucose levels of less than $12.2 \mathrm{mmol} / \mathrm{l}$ as unknown. An additional subject whose diabetes is controlled by diet with age at onset of 80 years and who was unavailable for testing was also classified as unknown. Similarly, although impaired glucose tolerance (IGT) in young individuals is likely to be a manifestation of a genetic predisposition to Type 2 diabetes, up to $25 \%$ of older individuals are IGT by non-age corrected WHO or National Diabetes Data Group criteria [13]. The standard WHO criteria for IGT (7.8 mmol/l) only exceeds the $95 \%$ level for a 2 -h-stimulated glucose for individuals under age 45 years. We thus performed a second analysis in which we considered individuals whose glucose levels exceeded these $95 \%$ criteria to be affected. All other individuals who met WHO criteria for IGT were considered to be of unknown diabetic status.

\section{Marker determination}

All pedigree members were typed for both $5^{\prime}$ (GCK2) and $3^{\prime}$ (GCK1) microsatellite polymorphisms by polymerase chain reaction (PCR) with Taq polymerase (Perkin Elmer Cetus, Norwalk, Conn., USA). A single primer was end-labelled with $\gamma^{32} \mathrm{P}$ (Amer- sham, Arlington Heights, Ill., USA) with polynucleotide kinase [14]. Amplification conditions for the $3^{\prime}$ polymorphism are described elsewhere [15]. The $5^{\prime}$ microsatellite was amplified with primers CTGTGCCATGGTTATATAAGATAAG and AAACAGATACGCTTCATCCTGATTC. Amplification was carried out for 30 cycles at $94^{\circ} \mathrm{C}$ for $1 \mathrm{~min}, 60^{\circ} \mathrm{C}$ for $1 \mathrm{~min}$, and $72^{\circ} \mathrm{C}$ for $1 \mathrm{~min}$. Amplified samples were separated on $6.0 \%$ denaturing polyacrylamide gels. Samples from pedigree members were run in proximity and against known markers to ensure consistent scoring among members of the same pedigree. For both GCK1 and GCK2 microsatellites, rare alleles were renamed to one of the three more common classes for purposes of linkage analysis, so that only three total alleles were analysed for each marker. This reclassification greatly simplifies computational analysis. Since alleles were redefined with reference to each pedigree, in no case was the information for linkage analysis altered.

\section{Linkage analysis}

Although positive results were obtained in MODY under a dominant model with a low gene frequency [7], we considered the appropriate genetic model to be unknown in familial Type 2 diabetes. Simulation studies suggest that linkage can be detected even when the true model is unknown by testing a range of models [16] (S. C.Elbein, unpublished data). We tested both diagnostic schemes (IGT subjects were considered as unknown, and all individuals exceeding the $95 \%$ cutoff for 2 -h glucose were considered affected) under dominant and recessive models. For dominant models we tested a gene frequency of 0.02 , and for recessive models a disease gene frequency of 0.2 . We have previously demonstrated that small changes (3-5 fold) in gene frequency have little impact on the results in these pedigrees (S.C.Elbein unpublished data). Each individual was assigned to one of seven liability classes derived from age in decades at time of testing, except that all individuals under age 25 years were assigned to class 1 , and all individuals over age 75 years were assigned to class 7 . We assigned an age-dependent linear penetrance function which increased in equal stepwise increments from 0.05 in class 1 to a maximum of 0.9 for class 7 . We similarly allowed for age-dependent phenocopies (non-inherited cases of diabetes) to a maximum of $2 \%$ prevalence (class 7 ) in non-susceptible genotypes. This factor permits one-third of all cases to result from an unlinked locus or nongenetic factors. In addition to these models, we tested the hypothesis suggested from MODY studies [7] that glucokinase defects represented a rare dominant disease. For these analyses, we tested a gene frequency of 0.002 and a maxiumum phenocopy frequency to 0.04 , such that most Type 2 diabetic patients were phenocopies. Finally, we examined a dominant model in which penetrance was 0.01 for class 1 and increased to only 0.5 maximum for class 7 (age older than 75 years). With low penetrance, unaffected individuals contributed little to the LOD score, and linkage might be detected which would be rejected by our other analyses if the higher penetrance function were incorrect [17]. All analyses were run using the MLINK program of the LINKAGE package (version $5.1 ;[18]$ ) on a $386 \mathrm{PC}$. AIlele frequencies were estimated from unrelated individuals in our population. Recombination between GCK1 and GCK2 was assumed to be 0 , and two point analysis between loci confirmed this assumption.

The hypothesis of linkage with heterogeneity was tested with the "A test" for heterogeneity performed on the results of the linkage analysis with the HOMOG program [18]. In this test, the proportion of linked families $(\alpha)$ is allowed to vary from 0 to 1 , and the likelihoods of heterogeneity and linkage under heterogeneity are examined. The statistic is distributed as a chi-square distribution. Although in the absence of linkage the appropriate significance level is uncertain, Ott has suggested a value of $p<0.001$ [18].

Simulations of linkage were performed for pedigree 9 to determine how closely our reported log of the odds (LOD) score approached the maximum attainable for this pedigree, and to determine the likelihood that a LOD score this high would be achieved by 
Table 1. Total log of the odds (LOD) scores for glucokinase vs familial Type 2 diabetes by genetic model

\begin{tabular}{lllll}
\hline $\begin{array}{l}\text { Mode of } \\
\text { inheritance }\end{array}$ & $\begin{array}{l}\text { Diagnostic } \\
\text { criteria }\end{array}$ & $\begin{array}{l}\text { Gene fre- } \\
\text { quency }\end{array}$ & $\begin{array}{l}\text { Phenocopy } \\
\text { frequency }\end{array}$ & $\begin{array}{l}\text { LOD score } \\
(=0.001)\end{array}$ \\
\hline Dominant & IGT unknown & 0.02 & 0.02 & -2.76 \\
Dominant & IGT unknown & 0.002 & 0.04 & -2.64 \\
Dominant & 95\% Cutoff & 0.02 & 0.02 & -2.59 \\
Dominant & 95\% Cutoff & 0.002 & 0.04 & -2.87 \\
Recessive & IGT unknown & 0.20 & 0.02 & -1.91 \\
Recessive & 95\% Cutoff & 0.20 & 0.02 & -1.13 \\
\hline
\end{tabular}

Total LOD scores are shown for the entire pedigree cohort for several models tested. Models are described in detail in Subjects and Methods. All Type 1 diabetic subjects were considered unknown, and all Type 2 diabetic subjects under treatment were considered affected. For "IGT unknown" models all individuals with impaired glucose tolerance (IGT) individuals or Type 2 diabetes diagnosed only by glucose tolerance test and whose 2 -h values during an oral glucose tolerance test did not exceed $95 \%$ age- and race-specific values were considered unknown. For " $95 \%$ cutoff", individuals were also considered affected if they exceeded these $95 \%$ age-specific population glucose levels; otherwise untreated individuals meeting WHO criteria for IGT or Type 2 diabetes were considered unknown. Phenocopy frequency represents the maximum value for an age adjusted rate of diabetes in non-susceptible genotypes

chance alone. Simulations were performed with the program SLINK $[18,19]$ and simulated files were analysed under MSIM, a modification of the MLINK program with quadratic interpolation to estimate the maximum expected LOD score [18].

\section{Results}

Linkage of glucokinase and Type 2 diabetes was investigated under both diagnostic schemes (IGT were considered as unknown, or all individuals with 2-h stimulated glucose levels in excess of the age-specific $95 \%$ cutoff level were considered affected) for dominant and recessive models. In linkage analysis of a candidate locus, one compares the likelihood of two hypotheses: that the Type 2 diabetes locus resides at the glucokinase locus, and thus diabetes and GCK are tightly linked (no recombination or recombination rate $\theta=0$ ) vs no linkage (random assortment or recombination rate $\theta=0.5$ ). The probability of linkage is expressed as the logarithm of the odds ratio (LOD score) for these hypotheses. Linkage is traditionally accepted when the LOD score exceeds 3 , or 1000:1 odds in favour of linkage, and is rejected when the LOD score is less than -2 , or 100:1 odds against linkage [18]. Our results are all reported at a recombination fraction of $\theta=0.001$, and are summarized in Table 1 . The least negative LOD scores were obtained under recessive models, in which LOD scores were in the indeterminate range of -1 to -2 . Linkage was clearly rejected (LOD less than -2 ) at a recombination fraction of $\theta=0.001$ under dominant models with both classification schemes. Linkage was also rejected $(\mathrm{LOD}=-2.87)$ when examined with a gene frequency of 0.002 and a high phenocopy frequency (to 0.04 maximum), such that most cases of Type 2 diabetic patients would represent phenocopies. We were unable to reject linkage under the less informative analysis with a low penetrance, however $(\mathrm{LOD}=-1.19)$.
Under each model, inspection of individual pedigree LOD scores showed that nearly one-third of pedigrees were uninformative (LOD very near 0 ), but pedigree 9 approached a LOD score of 2 under dominant models when young IGT individuals were considered affected, even with the low penetrance model ( $\mathrm{LOD}=1.82)$. Individual LOD scores under the dominant model with gene frequencies of 0.02 and 0.002 are shown in Table 2, and the segregation of GCK alleles for pedigree 9 is shown in Figure 1. In all cases, analysis at recombination fractions greater than 0.01 were less informative (closer to 0 ).

Because inspection of individual LOD scores suggested heterogeneity, we examined the hypothesis that Type 2 diabetes and GCK were linked in a subgroup of pedigrees. Initially we performed the "A test" for heterogeneity with the HOMOG program [18], as described in Subjects and Methods. We found no evidence for heterogeneity under recessive models. Under dominant models chi-square values $(2 d f)$ were $4.5-4.9(p<0.10)$ in support of linkage and heterogeneity. The likelihood was maximum when the proportion of linked pedigrees $(\alpha)$ was 0.2 to 0.3 , but significance was well below that proposed ( $p=0.001$ ) when the total LOD score under homogeneity is less than 3 [18].

To further investigate the possibility of linkage and heterogeneity, we tested the likelihood that we would obtain an LOD score of 1.9 by chance alone in pedigree 9 given these markers. Simulations were performed under a single dominant model with diagnostic criteria based on

Table 2. Individual pedigree $\log$ of the odds (LOD) scores at $\theta=0.001$ for dominant models

\begin{tabular}{lllrr}
\hline Pedigree & $\begin{array}{l}\text { Total number } \\
\text { of individuals } \\
\text { tested }\end{array}$ & $\begin{array}{l}\text { Total number } \\
\text { of affected } \\
\text { individuals }\end{array}$ & $\begin{array}{l}\text { LOD } \\
\text { score for } \\
\mathrm{q}=0.002\end{array}$ & $\begin{array}{l}\text { LOD } \\
\text { score for } \\
\mathrm{q}=0.02\end{array}$ \\
\hline 1 & 26 & 8 & -1.21 & -0.93 \\
2 & 23 & 4 & -0.93 & -0.75 \\
4 & 19 & 4 & -0.56 & -0.59 \\
5 & 18 & 4 & 0.02 & -0.03 \\
6 & 19 & 3 & -0.02 & -0.39 \\
7 & 29 & 4 & -0.94 & -1.14 \\
8 & 15 & 3 & 0.06 & 0.26 \\
9 & 25 & 7 & 1.99 & 1.93 \\
10 & 19 & 2 & 0.03 & 0.21 \\
11 & 20 & 4 & 0.61 & 0.72 \\
13 & 20 & 5 & -0.13 & -0.45 \\
14 & 20 & 6 & 0.12 & 0.14 \\
15 & 29 & 7 & 0.14 & 0.04 \\
16 & 26 & 7 & 0.15 & -0.01 \\
17 & 37 & 7 & -0.46 & -0.45 \\
18 & 14 & 5 & -1.16 & -0.49 \\
19 & 16 & 4 & -0.53 & -0.70 \\
20 & 20 & 5 & 0.07 & 0.04 \\
\hline
\end{tabular}

LOD scores are shown for each pedigree at $\theta=0.001$ for two models: dominant with a low gene frequency $(q=0.002)$ and high phenocopy frequency ( 0.04 maximum), as might be expected if glucokinase gene defects represent a rare dominant disease, and a gene frequency to account for a $4 \%$ disease prevalence $(q=0.02)$ with a phenocopy frequency to account for the remaining $6 \%$ disease prevalence (maximum 0.02). For both models shown, individuals were considered affected if they both met WHO criteria for impaired glucose tolerance and exceeded $95 \%$ age-specific population levels for 2 -h stimulated glucose (see Subjects and Methods and Table 1) 


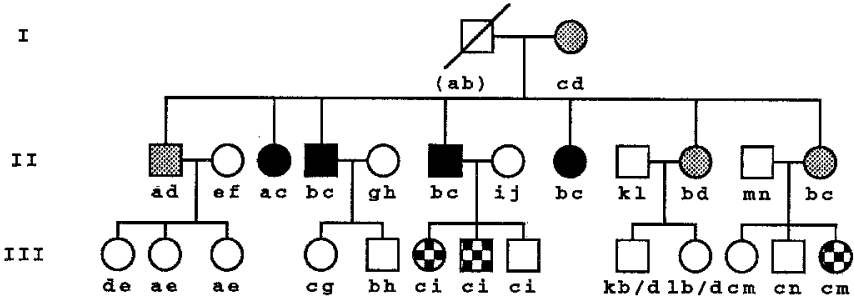

Fig.1. Glucokinase haplotypes for Pedigree 9. Glucokinase alleles, as determined by GCK1 and GCK2 haplotypes, are shown under the symbols on the pedigree. Blackened symbols denote Type 2 diabetic individuals, stippled symbols denote individuals with impaired glucose tolerance by WHO criteria, but whose stimulated glucose values did not exceed the $95 \%$ cutoff. These individuals had "unknown" disease status in linkage analysis. Checkered symbols represent individuals with impaired glucose tolerance whose stimulated glucose values exceeded age- and race-specific $95 \%$ values (see Subjects and Methods), and who were considered "affected" under appropriate models. Where unambiguous haplotypes could not be established (alleles were not distinguishable in offspring), both possibilities are shown with a slash separating them. Allele c segregated with diabetes in this pedigree

$95 \%$ levels for the 2-h glucose, with a gene frequency of 0.002 , and with a phenocopy rate of 0.04 maximum. To simplify analysis, we assumed complete linkage equilibrium between the GCK1 and GCK 2 markers and we combined rare alleles to give a single 6 allele locus (allele frequencies $0.47,0.26,0.10,0.06,0.06$ and 0.05$)$. The highest LOD score obtained from these simulations was 2.39. Because the assumption of linkage equilibrium overestimates the information for these polymorphisms (Y.Tanizawa, K. Chiu, unpublished data), this expected LOD score is higher than could be attained in reality, and our actual results thus approach the maximum LOD score possible in this single pedigree. In contrast, when we performed the same simulation experiment in the absence of true linkage $(\theta=0.5)$, a LOD score of 1.5 was exceeded in only 2 of 100 replicates (maximum 1.82).

To increase the power to detect linkage in the presence of heterogeneity, investigators have explored alternative methods to detect linkage. Age of onset has been a powerful means to rank pedigrees for this analysis [20], and the sequential addition of LOD scores actually corresponds to Morton's original description of linkage analysis [21]. Furthermore, data from MODY pedigrees suggested that glucokinase defects might lead to early-onset disease [7]. When we performed this analysis under a dominant model with pedigrees ranked in order of mean age of onset, the LOD score reached the maximum at 2.26 with the three youngest onset pedigrees, and then fell toward the values in Table 1 . This maximum was still below the 3.0 necessary to prove linkage under heterogeneity (linkage in an early-onset subgroup).

\section{Discussion}

The role of glucokinase in young-onset subsets of Type 2 diabetes (maturity-onset diabetes of the young, or MODY) is well-established [7, 9]. A role in more typical
Type 2 diabetes has been suggested by association studies [10], but GCK had not been examined in common familial Type 2 diabetic pedigrees. Our studies do not support a major role for GCK in these pedigrees. Under most models we significantly reject linkage. In simulation studies with this population using a single marker with comparable information content (heterozygosity 0.75 ), $70 \%$ of replicates hadLOD scores which exceeded 3 under homogeneous models, and $80 \%$ of LOD scores exceeded 1 even in the presence of $30 \%$ unlinked pedigrees. Thus, the LOD scores obtained in this study would be very unlikely if glucokinase defects were a major cause of Type 2 diabetes.

Our results do not exclude the possibility that glucokinase defects account for a small subset of Type 2 diabetic pedigrees. Indeed, in a single pedigree our LOD score approaches 2 , nearly the maximum which can be expected for that single pedigree. Although this finding is within the realm of chance, particularly since several models were tested, the results suggested a need for further evaluation. We have examined the clinical characteristics of this pedigree. Affected pedigree members ranked 2 and 3 for mean age of onset and percent desired body weight, respectively, among our pedigrees. These findings are consistent with the young age of onset in MODY pedigrees with GCK defects. However, we were unable to distinguish pedigrees with positive LOD scores from those with negative LOD scores by fasting or stimulated insulin levels. Furthermore, putative carriers of a GCK mutation in pedigree 9 had much higher insulin levels (after correction for age, sex, and weight) than did non-carriers (data not shown). Furthermore, sequence analysis of the nine exons for beta-cell glucokinase in the implicated allele has detected only silent mutations (S.C.Elbein unpublished data). Thus, the role of GCK mutations even in this pedigree is uncertain.

Our results point out a dilemma in the analysis of common and potentially heterogeneous disease. Linkage analysis is a powerful tool to test the hypothesis that a given locus predisposes to diabetes in the majority of pedigrees tested. In this respect linkage analysis has advantages over direct sequencing methods, since mutations in non-coding and regulatory regions are also detected. However, linkage analysis can at most suggest the involvement of genes which contribute to only $10 \%-20 \%$ of disease predisposition. This problem is compounded for markers which are not fully informative, such as in the present study. Thus, approximately $30 \%$ of pedigrees in our study were uninformative for linkage (LOD scores near 0). Furthermore, the Type 2 diabetic phenotype may result from the interaction of GCK mutations with other loci [4] or environmental factors. In that case, the actual penetrance for the GCK gene might be much lower than the $90 \%$ maximum which we used in most models. We have also examined a reduced penetrance model ( $50 \%$ maximum penetrance), and found no support for linkage under this model either. Nonetheless, reduced penetrance also diminishes the power to detect linkage, particularly if GCK mutations are present in only a fraction of pedigrees. Thus, linkage studies have little power to detect loci which are not major contributors to the disease susceptibility. 
Direct sequence analysis of coding regions provides an alternative method to identify mutations in the face of extreme heterogeneity or multiple interacting loci. However, in our examination of the nine glucokinase exons by single strand conformation polymorphism analysis $[22$, 23], we have not identified a mutation which segregates with Type 2 diabetes in these pedigrees (S.C. Elbein, unpublished data). Nonetheless, a predisposing mutation may reside in a regulatory region or an intron. Furthermore, screening methods do not have $100 \%$ sensitivity. Thus, we can clearly exclude a major role for glucokinase in familial Type 2 diabetes, but a thorough sequence analysis, particularly in pedigrees with positive LOD scores, may be necessary to totally exclude glucokinase mutations as a cause of familial Type 2 diabetes in this population.

Acknowledgements. This work was supported by grant National Institutes of Health Grand DK-39311 (SCE), and in part by National Institutes of Health grant DK-16746 (MAP) and the Research Service of the Veterans Affairs Medical Center (SCE).

\section{References}

1. Warram JH, Martin BC, Krolewski AS (1990) Slow glucose removal rate and hyperinsulinemia precede the development of type II diabetes in the offspring of diabetic parents. Annals Int Med 113: 909-915

2. Bergman RN (1989) Toward a physiological understanding of glucose tolerance: minimal model approach. Diabetes 38: 15121527

3. DeFronzo RA, Bonadonna RC, Ferrannini E (1992) Pathogenesis of NIDDM: a balanced overview. Diabetes Care 15:318-355

4. Rich SS (1990) Mapping genes in diabetes: genetic epidemiological perspective. Diabetes 39: 1315-1319

5. Magnuson MA (1990) Glucokinase gene structure: functional implications of molecular genetic studies. Diabetes 39: 523-527

6. Matchinsky FM (1990) Glucokinase as glucose sensor and metabolic signal generator in pancreatic $\beta$-cells and hepatocytes. Diabetes 39: 647-652

7. Froguel P, Vaxillaire M, Sun F et al. (1992) The glucokinase locus on chromosome $7 \mathrm{p}$ is closely linked to early onset non-insulindependent diabetes mellitus. Nature 356: 162-164

8. Vionnet N, Stoffel M, Takeda J et al. (1992) Nonsense mutation in the glucokinase gene causes early-onset non-insulin-dependent diabetes. Nature 356:721-722
9. Hattersley AT, Turner RC, Permutt MA et al. (1992) Linkage of type 2 diabetes to the glucokinase gene. Lancet $339: 1307-1310$

10. Chiu KC, Province MA, Permutt MA (1992) The glucokinase gene is a genetic marker for non-insulin dependent diabetes mellitus in American Blacks. Diabetes 41: 843-849

11. Elbein SC, Maxwell TM, Schumacher MC (1991) Insulin and glucose levels and prevalence of glucose intolerance in pedigrees with multiple diabetic siblings. Diabetes 40: 1024-1032

12. Elbein SC, Sorensen LK, Taylor M (1992) Linkage analysis of insulin-receptor gene in familial NIDDM. Diabetes 41: 648-656

13. Diabetes in America: diabetes data compiled 1984 (1985) Harris MI, Hamman RF (eds) U.S. Department of Health and Human Services, NIH Publ 85-1468, Washington, D.C.

14. Sambrook J, Fritsch EF, Maniatas T (1989) Molecular cloning: a laboratory manual. Cold Spring Harbor Laboratory Press, Cold Spring Harbor

15. Matsutani A, Janssen R, Donis-Keller H, Permutt MA (1992) A polymorphic $(\mathrm{CA})_{n}$ repeat element maps the human glucokinase (GCK) gene to chromosome 7p. Genomics 12: 319-325

16. Cox NJ, Hodge SE, Marazita ML, Spence MA, Kidd KK (1988) Some effects of selection strategies on linkage analysis. Genetic Epid 5: 289-297

17. Pericak-Vance MA, Bebout JL, Gaskell PC et al. (1991) Linkage studies in familial Alzheimer disease: evidence for chromosome 19 linkage. Am J Hum Genet 48: 1034-1050

18. Ott J (1991) Analysis of human genetic linkage, revised edition. Johns Hopkins University Press, Baltimore

19. Weeks DE, Ott J, Lathrop GM (1990) SLINK: a general simulation program for linkage analysis. Am J Hum Genet 47 [Suppl]: A204

20. Hall JM, Lee MK, Newman B et al. (1990) Linkage of early onset familial breast cancer to chromosome 17q21. Science 231: 1684 1689

21. Morton NE (1955) Sequential tests for the detection of linkage. Am J Hum Genet 7: 277-318

22. Orita M, Suzuki Y, Sekiya T, Hayashi K (1989) Rapid and sensitive detection of point mutations and DNA polymorphisms using polymerase chain reaction. Genomics 5: 874-879

23. Elbein SC, Sorensen LK (1991) Genetic variation in insulin receptor $\beta$-chain exons among members of familial type 2 (non-insulin-dependent) diabetic pedigrees. Diabetologia 34: 742-749

Received: 30 June 1992

and in revised form: 2 October 1992

Dr. S.C. Elbein

Division of Endocrinology

Veterans Affairs Medical Center 111E

500 Foothill Blvd.

Salt Lake City, UT 84148

USA 